QUARTERLY OF APPLIED MATHEMATICS

VOLUME L

SEPTEMBER $\cdot 1992$

NUMBER 3

SEPTEMBER 1992, PAGES 401-414

\title{
SELF-SIMILAR SOLUTIONS OF THE SECOND KIND OF NONLINEAR DIFFUSION-TYPE EQUATIONS
}

\author{
BY \\ JAVIER ALBERTO DIEZ \\ (Universidad Nacional del Centro de Provincia de Buenos Aires, Tandil, Argentina) \\ JULIO GRATTON (International Centre for Theoretical Physics, Trieste, Italy) \\ AND \\ FERNANDO MINOTTI (Universidad de Buenos Aires, Buenos Aires, Argentina)
}

\begin{abstract}
We study the self-similar solutions of the problem of one-dimensional nonlinear diffusion of a passive scalar $u$ (diffusivity $D \propto u^{m}, m \geq 1$ ) towards the centre of a cylindrical or spherical symmetry. It is shown that this problem has a self-similar solution of the second kind. The self-similarity exponent $\delta$ is found by solving a nonlinear eigenvalue problem arising from the requirement that the integral curve that represents the solution must join the appropriate singular points in the phase plane of the diffusion equation. In this way the integral curves that describe the solution before and after the diffusive current arrives at the centre of symmetry can be determined. The eigenvalues for different values of the nonlinearity index $m$ and for cylindrical and spherical geometry are computed. Numerical integration of the equations allows us to determine the shape of the solution in terms of the physical variables. The application to the case $m=3$, corresponding (for cylindrical symmetry) to the creeping gravity currents of a very viscous liquid, is worked out in detail.
\end{abstract}

1. Introduction. In this paper we shall be concerned with nonlinear diffusion equations of the type

$$
\frac{\partial u}{\partial t}=\nabla[D(u) \nabla u]
$$

in which the diffusivity $D \propto u^{m} \quad(m \geq 1)$. This equation is of considerable importance in mathematical physics, as it governs a variety of phenomena of different kinds. We mention the following examples:

(1) Flow in thin saturated regions in porous media in the Dupuit-Forchheimer approximation $(m=1)$ (see, for example, $[1,2,3])$.

Received September 21, 1989.

The first and third authors are Fellows of the Consejo Nacional de Investigaciones Cientificas y Técnicas. The second author is a Researcher of the Consejo Nacional de Investigaciones Cientificas y Técnicas. Permanent address: LPF, Facultad de Ciencias Exactas y Naturales, Universidad de Buenos Aires, Pabellon I, Ciudad Universitaria, 1428 Buenos Aires, Argentina.

(C)1992 Brown University 
(2) Percolation of a compressible polytropic gas in a porous medium $(m=\gamma=$ $c_{p} / c_{v} \geq 1 ;$ see $\left.[4,5,6]\right)$.

(3) Heat conduction by electrons in a plasma $(m=5 / 2$, see [7]).

(4) Viscous gravity currents $(m=3$, see $[8,9,10])$.

(5) Heat conduction by radiation in a multiply ionized gas $(m=4.5-5.5$, see $[7,11])$.

(6) Heat conduction by radiation in a fully ionized gas (Marshak waves, $m=$ $13 / 2$, see $[12,7,13])$.

We shall consider only one-dimensional problems, so that Eq. (1) takes the form

$$
\frac{\partial u}{\partial t}=x^{-n} \frac{\partial}{\partial x}\left(x^{n} u^{m} \frac{\partial u}{\partial x}\right) \text {, }
$$

in which $x$ denotes the spatial coordinate, and $n=0,1,2$ for plane, cylindrical, and spherical symmetry, respectively.

As is well known, Eq. (2) admits a self-similar solution if the problem at hand involves no more than one parameter, say $b$, with independent dimensions, $[b]=$ $L T^{-\delta}$. The numerical constant $\delta$ is the self-similarity exponent. The similarity solutions of nonlinear diffusion-type equations have been studied by many authors, including Barenblatt [14], Barenblatt and Zel'dovich [15], Pattle [16], etc., and more recently by Pert [11], Grundy [17], Huppert [9], and Gratton and Minotti [10], where more references can be found. With few exceptions all the solutions discussed in the literature belong to what is called self-similarity of the first kind, in which the exponent $\delta$ is determined by dimensional analysis in terms of the constant dimensional parameters of the problem.

Among the various self-similar solutions of Eq. (2), we shall investigate here those corresponding to a particular class of problems that lead to self-similar solutions of the second kind. In these cases the solution cannot be found by means of dimensional analysis, nor by the application of conservation laws (see $[7,18]$ in which these matters are discussed in detail). The self-similar solutions of the second kind can be obtained by any of the following procedures: (a) by starting from an adequate non-self-similar problem, and following its solution (either numerically, or in an experiment) until the self-similar asymptotics is approached, (b) by direct construction, requiring the existence at large of the desired self-similar solution of Eq. (2). In the last case, one is led to a nonlinear eigenvalue problem that allows us to determine the solution. This is the approach that shall be followed in the present paper.

A particular example of this type of self-similar solutions of the second kind was first obtained by Gratton and Minotti [10] in the course of an investigation on viscous gravity currents, and corresponds to $m=3$ and $n=1$ (axial symmetry) in (2). We shall take this example as the type case in order to keep the discussion as concrete as possible. It should be mentioned that this problem has previously been considered by D. G. Aronson [19] in a rather sketchy survey, where a mathematical proof of the existence of the solution for general $m>1$ is outlined; also a numerical solution was computed by Kevrekidies [20]. However, considering the interest of the subject it is justified to investigate in detail the spectrum of eigenvalues and the properties of the solutions. 
It must be kept in mind that the results of this paper are of a general nature, and can be applied, mutatis mutandis, to any of the phenomena described above, and also to spherical symmetry (that for viscous gravity currents cannot be realized). Accordingly, in what follows the term "current" must be understood in a generalized sense, i.e., as meaning any of the phenomena described by Eq. (1), if not otherwise indicated. To our best knowledge the family of self-similar solutions of the second kind representing converging diffusive currents had so far escaped detection (with the above-mentioned exception). Clearly its investigation is of considerable theoretical interest in view of the wide range of applications.

2. The collapse of a converging current: Self-similarity of the second kind. The type case we shall discuss is that of an axisymmetric gravity current of a very viscous liquid over a rigid horizontal surface which converges towards the origin. This type of current can be produced, for example, if there is initially a pool of liquid surrounding a circular wall; the liquid extends to infinity on the outside of the wall, while the circular inner part of the supporting surface is dry. If at a certain moment the retaining wall is removed, a gravity current towards the centre (the origin) will ensue. This current will have a converging front, whose radius will decrease with the passing of time, so that the front ultimately collapses at the origin.

Using the lubrication approximation (for discussions of this model, in which it is assumed a nearly horizontal motion governed by a balance between the forces due to gravity and those due to viscosity and inertia is neglected, see $[8,9,10])$ the flow is described by Eq. (2), in which $u=(g / 3 \nu)^{1 / 3} H \quad(H(x, t)$ denotes the thickness of the current, $g$ is the acceleration of gravity, $\nu$ is the cinematic viscosity coefficient), and $m=3, n=1$ as already said.

We shall be interested in the behaviour of the current near the instant of collapse, which we shall assume occurs at $t=0$ (thus $t<0$ corresponds to times previous to the collapse, when the converging front is approaching the origin, and $t>0$ to times after the collapse, when the current has covered the initially dry region, but there is still flow tending to increase the thickness of the liquid in the central part). We shall only be interested in the properties of the current near the front, or, equivalently, in its central part, i.e., for radii negligibly small as compared with any parameter that characterizes the initial depth of the pool outside the circular wall.

In this situation we are left with no constant dimensional governing parameter: those arising from the initial conditions are no longer adequate as a scale of the properties of the current in the region of interest, and the characteristic parameters of the flow are functions of time. Therefore the flow is self-similar, but the selfsimilarity exponent cannot be determined by simple dimensional analysis (as it is not possible by these means to determine the parameter $b$ ). We are in the presence of self-similarity of the second kind.

The generalization of the present arguments to other types of converging diffusive currents characterized by different values of $m$ and $n$ can easily be obtained by analogy. Some examples will be discussed further on. It can be observed that these problems are strongly reminiscent of that of a converging shock wave in gas dynamics 
(see $[19,7]$ ), which also yields a self-similar asymptotics of the second kind under conditions of the same type as those discussed above.

3. Basic equations and phase plane formalism. A convenient method to find the self-similar solutions of Eq. (2) is based on a phase plane formalism, as discussed in [10]. In this reference, only the case $m=3, n=0,1$ has been discussed in detail. In view of the applications to the phenomena mentioned in the Introduction, we shall extend the analysis to arbitrary values of $m(\geq 0)$ and to any symmetry.

We introduce a new dependent variable, $v$, as

$$
v=-u^{m-1} \frac{\partial u}{\partial x}
$$

(in the case of the viscous gravity currents, $v$ represents the vertically averaged flow velocity). With this definition Eq. (2) is then equivalent to the following system of first-order partial differential equations:

$$
u^{m-1} \frac{\partial h}{\partial x}+v=0, \quad \frac{\partial u}{\partial t}+\frac{\partial}{\partial x}(u v)+\frac{n u v}{x}=0 .
$$

This system admits self-similar solutions of the form

$$
u=\left[x^{2} t^{-1} Z(\xi)\right]^{1 / m}, \quad v=x t^{-1} V(\xi), \quad \xi=x / b t^{\delta},
$$

where $V$ and $Z$ are usually called phase variables. Substituting (5) in (4) one obtains

$$
\xi Z^{\prime}+2 Z^{\prime}+m V^{\prime}=0, \quad m \xi Z V^{\prime}-\xi Z^{\prime}(\delta-V)+(\beta V-1) Z=0,
$$

where the primes denote derivatives with respect to $\xi$, and

$$
\beta=2+m(n+1) \text {. }
$$

As the independent variable appears in (6) only through logarithmic derivatives, it is possible to reduce this system to an autonomous first-order differential equation for $V(Z)$ :

$$
\frac{d V}{d Z}=\frac{Z(2 \delta-1)+m(n+1) V Z+m(\delta-V) V}{m Z(2 Z+m V)} .
$$

Once $(8)$ is solved, a simple quadrature allows us to compute $\xi$ :

$$
\frac{d}{d Z}(\ln |\xi|)=-\frac{1}{2 Z+m V}
$$

Therefore, for any given $\delta$, the solution of a self-similar problem is essentially reduced to the integration (usually numerical) of the autonomous equation (8). Once $V(Z)$ has been found one can determine $\xi(Z)$ using $(9)$, and finally $Z(\xi)$ and $V(\xi)$ by inversion.

The plane $(Z, V)$ is usually called the phase plane. A solution of $(8)$ is represented by an integral curve in the phase plane. A single curve represents a self-similar solution of some kind. The solution of a given self-similar problem characterized by certain particular boundary conditions is represented in the phase plane by one or more pieces (adequately joined) of the appropriate integral curves which satisfy at their ends the boundary conditions. Any piece represents the solution in a certain domain of the independent variable. 
To ascertain which integral curve corresponds to the problem under study (i.e., to the given initial and boundary conditions) it is essential to know the behaviour of the solutions in the neighborhood of the singular points of (8). A complete discussion of the singular points can be found in [10] for $m=3$, and is presented there in detail. Here we have extended the analysis for arbitrary $m$, and the main properties, the asymptotic behaviour of the solutions, and the corresponding physical interpretation. A collection of examples which show how to construct the explicit self-similar solutions for various problems can be found in the above-mentioned reference.

4. Construction of the self-similar solutions for converging currents. It was shown in Sec. 2 that the solutions of (2) representing converging currents in cylindrical and spherical symmetry have a self-similar intermediate asymptotics of the second kind. We shall now determine this asymptotics by direct construction, i.e., starting from (8) and (9), and requiring the existence of the solution at large.

Let us first consider the solution before collapse $(t<0)$. For negative time, the integral curve of interest must lie in the $Z<0$ half plane, since $u$ must be a positive quantity (see the first of Eqs. (5)). Clearly, the solution we are seeking must have a moving front at a finite distance $x_{f}$ from the origin. Then, the corresponding integral curve must start at the singular point $A\left(Z_{A}=0, V_{A}=\delta\right.$, see the Appendix $)$ which represents a moving front. Since $A$ is a saddle, there is a single integral curve passing through it (beside the curve $Z=0$, which represents a trivial uninteresting solution). The trajectory leaving $A$ must end at some other singular point, and, for $Z<0$, the only candidates are $B\left(Z_{B}=-m / 2 \beta, V_{B}=1 / \beta\right), O\left(Z_{0}=0, V_{O}=0\right)$, or $C$ $\left(Z_{C}=0, V_{C}= \pm \infty\right)$; the properties of the solutions near these singular points are given in the Appendix.

We shall now show that only a trajectory joining $A$ with $O$ has the desired properties. In fact, it can be verified that an integral curve going from $A$ to $B$ (or to $C)$ represents a current which, for $-t \rightarrow 0$, blows up $(u, v \rightarrow \infty)$ or vanishes $(u, v \rightarrow 0)$ at a finite distance from the origin. Then trajectories of these types cannot represent the required solution. On the other hand, an integral curve joining $A$ with $O$ represents a current with the correct properties $(u, v$ finite and nonzero at any finite distance behind the front for $-t \rightarrow 0$ ). Then our solution must be represented by a trajectory going from $A$ to $O$.

Now, it must be observed that for curves coming from $Z<0, O$ is a saddle; in other words, there is a single curve in this half plane that arrives at $O$. All other curves in the neighborhood must either go to $B$, or to $C$. Then for arbitrary $\delta$ there is no integral curve joining $A$ with $O$. Such a curve exists only if $\delta$ has a particular value, $\delta=\delta_{c}$. This critical value must be found numerically (see below). For the special case $n=0$ and arbitrary $m$ the solution is fully analytical and corresponds to $\delta=1$; it reads as: $Z=m V(V-1)$ (see Gratton and Minotti [10], for $m=3$ ). The existence of the solution for $n>0$ was first reported by Aronson [19], where the solution is explicity given.

We then see that the requirement of the existence of a self-similar solution leads to an eigenvalue problem for the self-similar exponent, as is characteristic of selfsimilarity of the second kind. 
After solving the eigenvalue problem it is possible to find the solution for $t>0$, i.e., after the collapse. It obviously correponds to $\delta=\delta_{c}$, it must represent a current having zero flow (flow $\propto x^{n} u v$ ) and a finite value of $u$ for $\xi=0$, and an incoming flow far from the origin of coordinates. Since $t>0$, the integral curve must lie in the $Z>0$ half plane. It can be verified that the appropriate integral curve starts at the singular point $D$ (representing the origin of coordinates; $Z_{D}=\infty$, $V_{D}=(1-2 \delta) / m(n+1)$ ) and ends at $O$ (which represents $\left.\xi \rightarrow \infty\right) ; D$ is a saddle, and there is a single integral curve leaving it that arrives at the finite in the phase plane; we omit the details for brevity.

5. Determination of the eigenvalue, integral curves, and properties of the solutions. We shall show first that the eigenvalue lies in the interval $1 / 2<\delta<1$. To this end we consider the behaviour of the solution for $\xi \rightarrow \infty$. According to the results reported in the Appendix, near $O$ one has approximately

$$
u \propto x^{(2 \delta-1) / m \delta}, \quad v \propto x^{(\delta-1) / \delta} .
$$

Therefore, if $v$ vanishes for $x \rightarrow \infty$ (i.e., the liquid should be at rest, or the heat flow should vanish, etc., according to the kind of phenomenon we are considering), then $\delta_{c}<1$. Furthermore, $u$ cannot decrease for $x \rightarrow \infty$, as this would mean that we have another front there, or a sink (of mass, or of heat), so that $1 / 2<\delta_{c}$. This lower bound can also be demonstrated observing that for $t>0$, and near the origin (this corresponds to the neighborhood of the singular point $D$, see the Appendix), $u$ must be an increasing function of time.

The eigenvalue can be found by a simple trial and error method. A trial value $\delta_{1}$ is assumed, and Eq. (8) is integrated numerically. Since it is not possible to integrate through the whole interval of interest, the following technique is employed to find $\delta_{c}$ : the integration is started from both $O$ and $A$, and continued on the two sides until the curves arrive, respectively, at the points $Z_{O, 1}$ and $Z_{A, 1}$ where they have a vertical slope. In general $Z_{O, 1}$ and $Z_{A, 1}$ will not coincide since $\delta_{c} \neq \delta_{1}$. Next, a second attempt is made, based on a new guess $\delta_{2}$, and new values $Z_{O, 2}$ and $Z_{A, 2}$ are determined. Then, using $Z_{O, i}$ and $Z_{A, i}(i=1,2)$ one interpolates (or extrapolates) linearly to obtain a new guess $\delta_{3}$ which will be a better approximation to $\delta_{c}$. This procedure can be repeated as many times as necessary to obtain the value of $\delta_{c}$ with the desired precision. The details of the computations can be found in [21]. In Table I (see p. 408) and Fig. 1 we give the values of $\delta_{c}$ for different values of the geometrical index $n$ and the nonlinearity index $m$. It should be mentioned that according to Aronson [19], J. Graveleau communicated to him that he had numerically determined the solution. The eigenvalue for $m=1$ and $n=1$ is given in this reference and coincides with the value determined in this paper.

The asymptotic formula for the integral curve near the point $A$ (the front), used to start the integration, is

$$
V=\delta+\frac{\beta \delta-1}{m(m+1)} Z+\cdots
$$




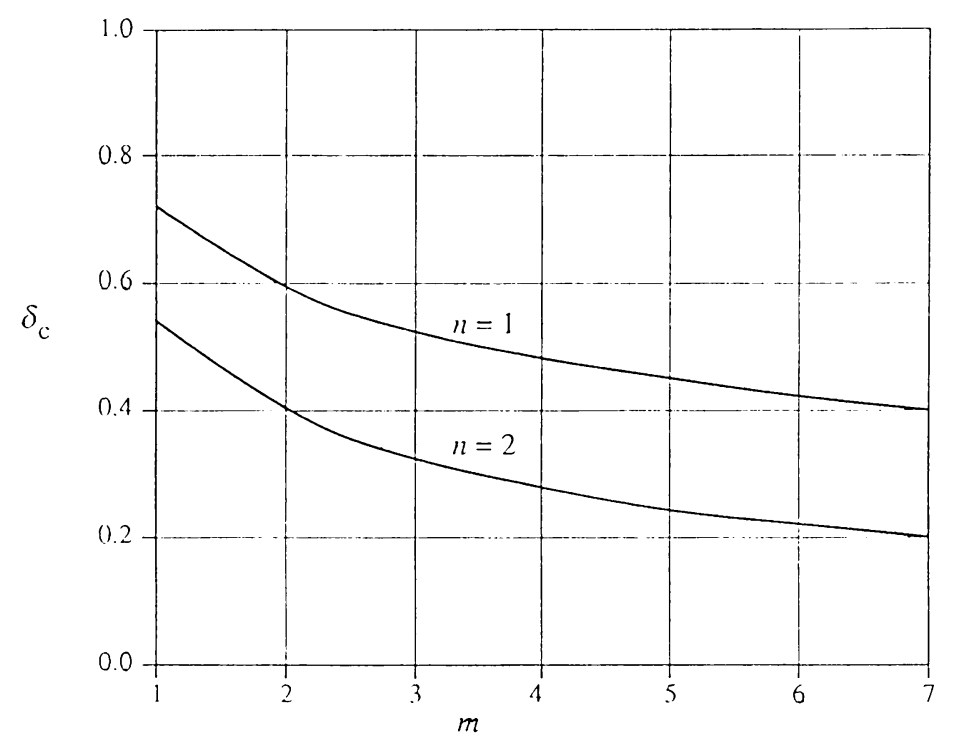

FIG. 1. Self-similar exponents (eigenvalues) as functions of the nonlinearity index $m$, for cylindrical $(n=1)$ and spherical $(n=2)$ symmetry.

The corresponding solution near the front is given by

$$
u=\left[\left(\frac{-m \delta x_{f}^{2}}{t}\right)(1-\eta)\right]^{1 / m}\left[1+\frac{(1+m n) \delta-1}{2 m(m+1) \delta}\right](1-\eta)+\cdots
$$

and

$$
v=\delta\left(\frac{x_{f}}{t}\right) \eta\left[1+\frac{\beta \delta-1}{(m+1) \delta}(1-\eta)+\cdots\right],
$$

where $\eta=x / x_{f}$ and $x_{f}=K(-t)^{\delta}, K=$ const. From these formulae it can be seen that as $-t \rightarrow 0$ the front accelerates, its speed tending to infinity at the moment of collapse.

All the integral curves that arrive at $O$ are given by

$$
V=-\frac{2 \delta-1}{m} Z\left\{1-\frac{(\beta \delta-1)(m+1)}{m \delta^{2}} Z\left[1-\left(\frac{(2 \delta-1)(3+2 m)}{m \delta}-3+n\right) Z\right]+\cdots\right\}
$$

For $Z>0$ there is an infinity of integral curves arriving at $O$; for $Z<0$, as already said, there is only one such line.

The integral curve that represents the solution after the collapse goes from $D$ to $O$, and can be obtained (once $\delta_{c}$ has been found) by numerical integration starting from $D$. Near $D$ this line is given approximately by

$$
V=-\frac{2 \delta-1}{m(n+1)}\left[1-\frac{\beta \delta-1}{m(n+1)(n+3) Z}\right] .
$$

It can be noticed that the self-similarity variable $\xi$ is determined within a constant numerical factor, since the eigenvalue $\delta_{c}$ fixes the dimensions of $b$, but not its 
Table I. Self-similar exponents $\delta_{c}$ for different values of the nonlinearity index $m$ and geometrical index $n$ ( $n=1$ for cylindrical, $n=2$ for spherical geometry).

\begin{tabular}{ccc}
\multicolumn{3}{c}{$\delta_{c}$} \\
\hline$m$ & $n=1$ & $n=2$ \\
\hline 1.0 & 0.856554 & 0.771452 \\
1.5 & 0.821558 & 0.728002 \\
2.0 & 0.796439 & 0.698553 \\
2.5 & 0.777286 & 0.677322 \\
3.0 & 0.762116 & 0.661124 \\
3.5 & 0.749698 & 0.648248 \\
4.0 & 0.739577 & 0.637777 \\
4.5 & 0.730486 & 0.629294 \\
5.0 & 0.722874 & 0.622229 \\
5.5 & 0.716192 & 0.615260 \\
6.0 & 0.710324 & 0.609715 \\
6.5 & 0.704921 & 0.604914 \\
7.0 & 0.700152 & 0.600678 \\
\hline
\end{tabular}

numerical value. This numerical value can only be obtained by actually performing an experiment, or by means of a numerical simulation of the complete non-self-similar problem, starting from adequate initial conditions, and following the calculations until the self-similar intermediate asymptotics is attained (see [18]).

The profiles of $u, v$ before and after collapse are represented in Figs. 2, 3 and 4, 5, respectively, for $m=3$, and $n=1,2$, as functions of $\eta=x / x_{f}$ (see pp. 409 and $410)$. In the problem of the circular wall $(m=3, n=1)$, the solution represents a viscous current whose height and velocity are proportional to $u$ and $v$, respectively. For other values of the nonlinearity index the shape of the profiles is similar, and only the numerical values are changed. In this way, the profiles of thickness and velocity of a liquid layer that flows in a saturated porous medium $(m=1, n=1)$ are similar to those represented in Figs. 2-5. 


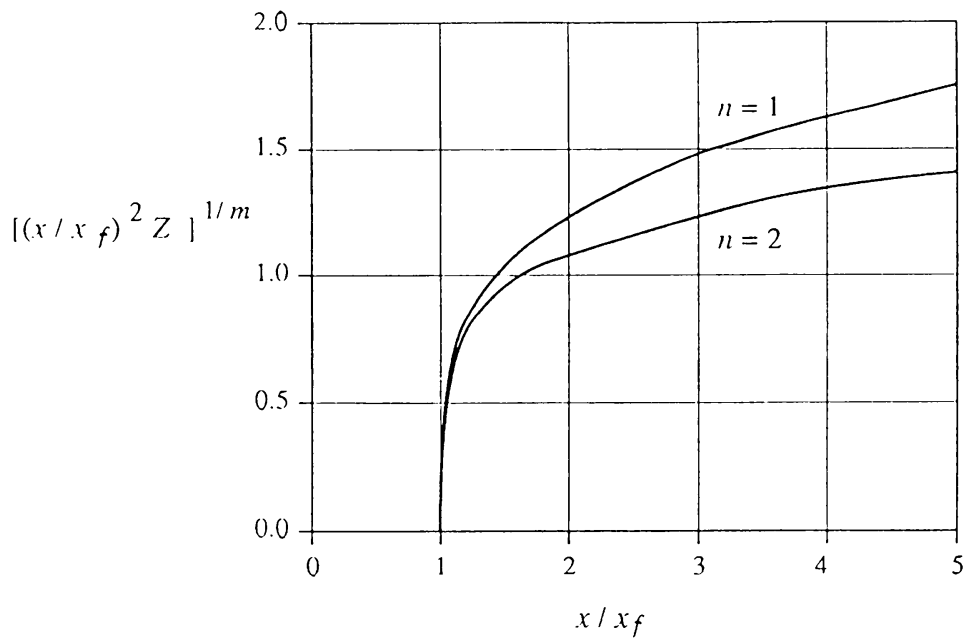

FIG. 2. Solutions before collapse $(t<0, m=3)$. Profiles of $u$.

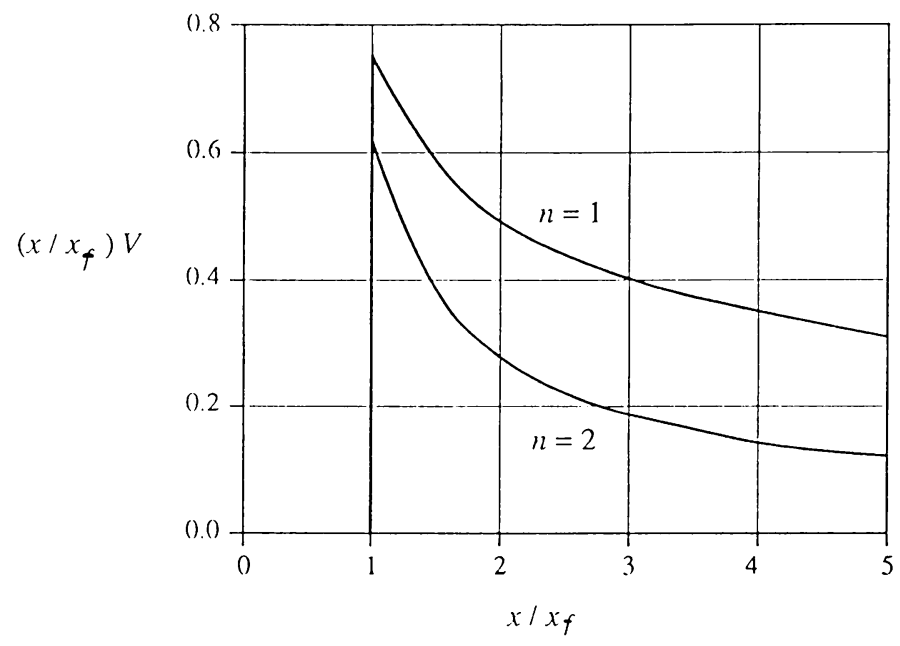

Fig. 3. Solutions before collapse $(t<0, m=3)$. Profiles of $|v|$.

The spherical symmetric case $(n=2)$ is of course meaningless in the abovementioned problems that deal with essentially two-dimensional currents, but is of interest for three-dimensional phenomena like the percolation of a polytropic gas in a porous medium $(m=\gamma \geq 1)$, and the nonlinear heat conduction (either by radiation or by electrons). In the first instance the physical problem is that of an infinite porous medium in which there is initially a spherical (or a cylindrical) region devoid of gas, which is being filled by the percolation of the gas from the surrounding regions. In the second case our solutions describe the asymptotics corresponding to an initial condition in which there is a spherical (or cylindrical) cold region, surrounded by a hot (temperature larger than, say, $10^{4}{ }^{\circ} \mathrm{K}$ ) region which extends to infinity; in this situation a converging thermal wave will travel into the inner cold region. 


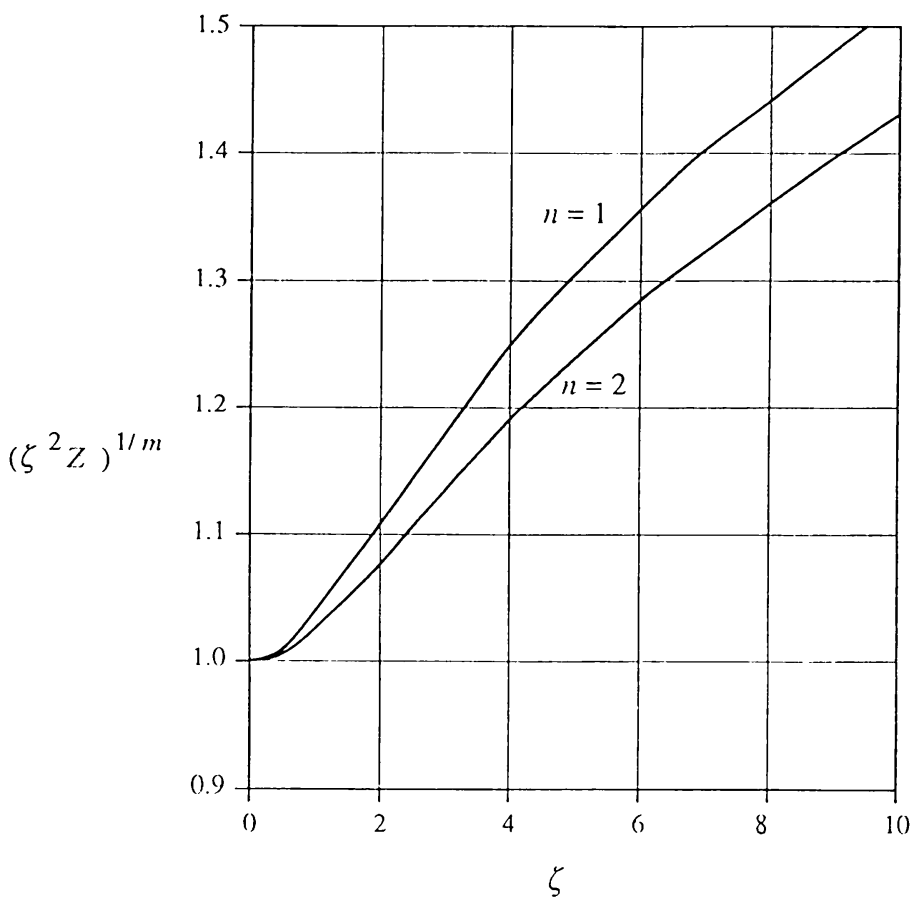

FIG. 4. Solutions after collapse $(t>0, m=3)$. Profiles of $u$.

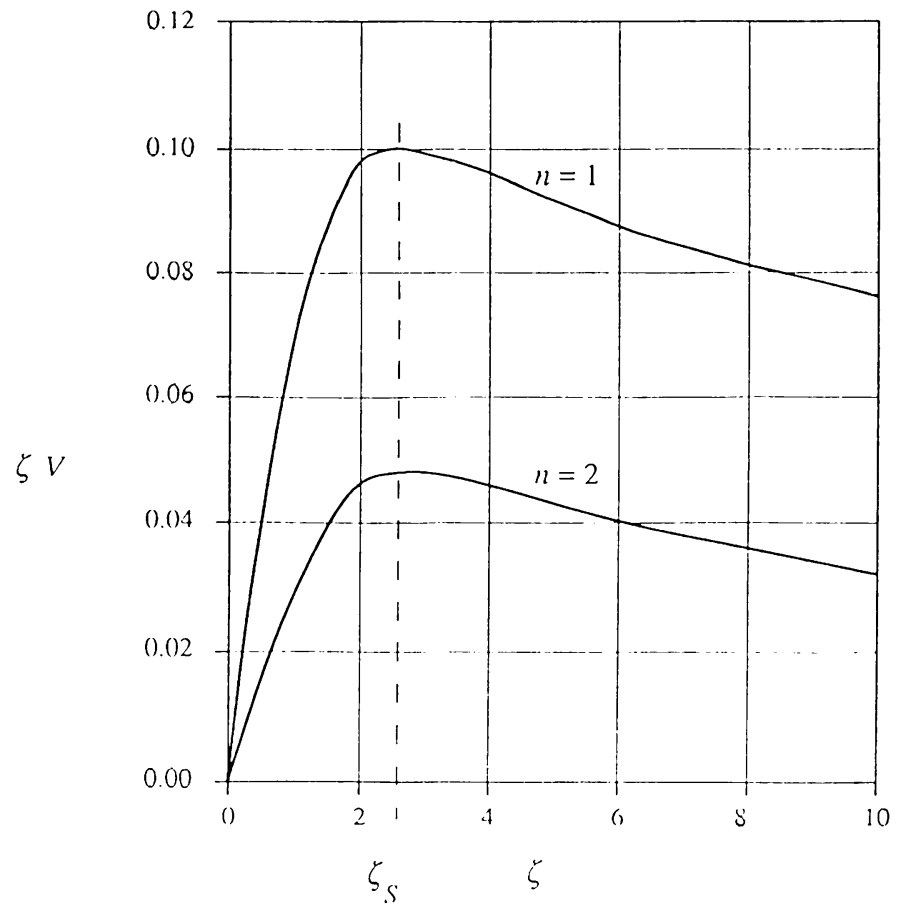

FIG. 5. Solutions after collapse $(t>0, m=3)$. Profiles of $|v|$. 
It can be observed that in this problem one can neglect the motion of the medium (partially ionized gas, or plasma) in which the heat propagates. In effect, let us consider, for simplicity, a gaseous medium; then a compression wave (or a shock wave) will be formed at the boundary between the hot and cold regions, and will travel into the cold unperturbed medium with a velocity of the order of the speed of sound in the hot region. The thermal wave initially has a velocity of the order $d x_{f} / d t \propto \sqrt{(D / t)}$, where $D \propto \vartheta^{m} \quad(\vartheta=$ temperature $)$. Then $x_{f} \propto \vartheta^{m / 2}$ for $t$ finite and nonzero. On the other hand, the speed of sound in a high temperature gas is roughly proportional to $\vartheta^{1 / 2}$. Consequently, if the temperature is very high, the initial velocity of propagation of the thermal wave will be much larger than the speed of sound. In addition, as the moment of collapse is approached $(-t \rightarrow 0)$, the velocity of the front of the thermal wave tends to infinity as $(-t)^{\delta-1}$. Then the propagation of the thermal front takes place in a medium at rest.

Let us now discuss briefly the behaviour of the solution after the collapse, illustrated by the profiles of Figs. 4-5. It can be seen that near the centre of symmetry $u \propto t^{(2 \delta-1) / m}$; of course this behaviour is limited to a short interval of time after the collapse; in fact, $u$ cannot increase indefinitely, but for large $t>0$ it must eventually approach a constant value. It is also interesting to observe that (a) as $t$ passes through zero, $v$ (the flow velocity, in the case of the viscous current), which before collapse is very large near the centre, becomes very small later on (for reasons of symmetry $v=0$ at the centre), and (b) for any fixed $t$, as $x$ is varied from large values to the origin, $v$ increases at first, passes through a maximum at a certain locus $S$ at a radius $x_{S}$, and then decreases to zero at the centre. The locus of maximum $v$ moves outwards with time according to $x_{S} \propto t^{\delta}$. This is due to the nonlinearity of the diffusion equation (1), one of whose effects is that perturbations are propagated with a finite speed. The locus $S$ carries outwards the information that the current has reached the centre of symmetry, so that the rate of flow (proportional to $v$ ) must diminish. The results obtained for different values of $m$ and $n$ show that the value of $\xi_{S}$ depends on $m$ but not on $n$.

6. Final remarks. We have studied one-dimensional currents of the nonlinear diffusive type in the last stages of convergence towards the centre of (cylindrical and spherical) symmetry, and in the first stages after the collapse of the current front. General values of the nonlinearity index are considered. For concreteness, the convergent viscous gravity current is taken as the type case and is discussed in detail. The analysis of the governing equations and parameters shows that the stages of interest follow a self-similar intermediate asymptotics of the second kind. This important type of self-similarity is well known in other problems of mathematical physics, but until now the self-similar asymptotics of the second kind of convergent nonlinear diffusion currents has not been discussed in the literature. The fact that the nonlinear diffusion equation describes a large number of interesting phenomena, as those mentioned in the Introduction, adds to relevance of the results we have derived. 
In the present paper we have put a certain emphasis on the solutions corresponding to the viscous gravity currents $(m=3, n=1)$, since the theoretical results obtained here can be verified by means of simple experiments which can be carried out with modest technical resources. In fact, as a continuation of this research, we are presently engaged in a series of experiments in which a convergent gravity current of a very viscous liquid is set up in a circular tank. The details will be published elsewhere; the results validate the present theory, with the limitations of its approximations [22].

Appendix. We summarize here some relevant properties of the singular points of Eq. (8), their physical interpretation, and the behaviour of the solution in their vicinity. The present results are an extension to arbitrary values of the nonlinearity index $m$ of those reported in [10] for the special case $m=3$. Other details of this particular case can be found in this reference.

The six singular points of (8) are denoted by $O, A, B, C, D$, and $E ; K$ denotes a numerical constant. The flow is defined as $F=\Pi x^{n} u v \quad(\Pi=1,2 \pi, 4 \pi$ for $n=0,1,2$, respectively). We also use the following notation:

$$
\begin{gathered}
\delta_{ \pm}=\delta_{0} \pm \lambda, \quad \delta_{0}=\frac{\beta+2(m+1)}{2 \beta}, \\
\lambda=\left\{\delta_{0}^{2}-[(\beta+2)(4 m+\beta+2)+4 m(m-2 \beta)] / 4 \beta^{2}\right\}^{1 / 2} .
\end{gathered}
$$

We examine below the different singular points.

1. $O\left(Z_{O}=0, V_{O}=0\right)$.

1.1. For $\delta=0, O$ is a cusp; it represents a front at a fixed position $x=x_{f}$. One has

$$
u \propto\left[-\left(x-x_{f}\right)^{2} / t\right]^{1 / m}, \quad v \propto\left(x-x_{f}\right) / t .
$$

1.2 For $\delta \neq 0, O$ is a saddle-node (saddle for the curves arriving from $Z<0$, and node for the curves arriving from $Z>0$ ); it represents $x=\infty$. Two cases must be distinguished:

1.2.1. $\delta \neq 1 / 2: F \neq 0$ at $x=\infty$, and

$$
u \propto x^{(2 \delta-1) / m \delta}, \quad v \propto x^{(\delta-1) / \delta}
$$

notice that $u$ and $v$ are independent of $\xi$.

1.2.2. $\delta=1 / 2: F=0$ at $x=\infty$, and

$$
u \propto \text { const. }, \quad v \propto(x / t) \exp \left(-K \xi^{2}\right) .
$$

2. $A\left(Z_{A}=0, V_{A}=\delta\right)$. Point $A$ is a saddle, representing a moving front $\left(x_{f} \propto t^{\delta}\right)$ with

$$
u \propto t^{(2 \delta-1) / m}\left(1-x / x_{f}\right)^{1 / m}, \quad v \propto x / t .
$$

3. $B\left(Z_{B}=-m / 2 \beta, V_{B}=-1 / \beta\right)$. The nature of this singular point varies with $\delta:$

(a) $B$ is a node for $0 \leq \delta \leq \delta_{-}$.

(b) $B$ is a focus for $\delta_{-}<\delta<\delta_{+}$.

(c) $B$ is a node for $\delta_{+}<\delta$. 
The physical interpretation also varies with $\delta$ :

3.1. For $\delta<\delta_{0}, B$ represents $x=\infty$, and $F \neq 0$.

3.2. For $\delta>\delta_{0}, B$ represents $x=0$. In both cases, one has

$$
u \propto\left(-x^{2} / t\right)^{1 / m}, \quad v \propto x / t .
$$

4. $C\left(Z_{C}=0, V_{C}= \pm \infty\right)$. Point $C$ is a node, representing a moving sink $\left(x_{f} \propto t^{\delta}\right)$ with

$$
u \propto t^{(2 \delta-1) / m}\left(1-x / x_{f}\right)^{1 /(m+1)}, \quad v \propto t^{\delta-1}\left(x / x_{f}\right)\left(1-x / x_{f}\right)^{-1 /(m+1)} .
$$

5. $D\left(Z_{D}=\infty, V_{D}=(1-2 \delta) / m(\alpha+1)\right)$. Point $D$ is a saddle, it represents $x=0$ with $F=0$; near $D$ :

$$
u \propto t^{(2 \delta-1) / m}, \quad v \propto x / t
$$

notice that $u$ is independent of $x$ (for $x$ near the origin).

6. $E\left(Z_{E}=\infty, V_{E}=\infty\right)$. This singular point is a saddle-node, it represents $x=0$. Two cases can be distinguished:

6.1. $n=0$ (plane symmetry); two types of behaviour are possible:

6.1.1. $x$-dependent behaviour, only $F>0$ :

$$
\begin{aligned}
& u \propto x^{1 /(m+1)} t^{[(m+2) \delta /(m+1)-1] / m}, \\
& v \propto x^{-1 /(m+1)} t^{(m+2) \delta /(m+1)-1} .
\end{aligned}
$$

6.1.2 $x$-independent:

$$
u \propto t^{(2 \delta-1) / m}, \quad v \propto t^{\delta-1} .
$$

6.2. $n \neq 0$; only $F>0$, and

$$
u \propto t^{(2 \delta-1) / m}|\ln \xi|^{1 /(m+1)}, \quad v \propto t^{2 \delta-1} x^{-1}|\ln \xi|^{-1 /(m+1)} .
$$

Acknowledgments. One of the authors (J.G.) thanks Prof. Abdus Salam and the International Centre for Theoretical Physics for their assistance. We also acknowledge grants of the Organization of American States, the Consejo Nacional de Investigaciones Cientificas y Técnicas, the Universidad de Buenos Aires, and the Universidad Nacional del Centro de la Provincia de Buenos Aires.

\section{REFERENCES}

[1] P. Y. Polubarinova-Kochina, Theory of Ground Water Movement, Princeton Univ. Press, Princeton, NJ, 1962

[2] P. S. Eagleson, Dynamic Hydrology, McGraw-Hill, New York, 1970

[3] L. A. Peletier, Applications of Nonlinear Analysis in the Physical Sciences, Chap. II: The porous media equation, Pitman Adv. Publ. Progr., Boston, 1981

[4] M. Muskat, The Flow of Homogeneous Fluids through Porous Media, McGraw-Hill, New York, 1937

[5] B. H. Gilding and L. A. Peletier, On a class of similarity solutions of the porous media equation, J. Math. Anal. Appl. 55, 351-364 (1976)

[6] J. L. Vazquez, Free Boundary Problems: Theory and Applications, Vol. I, Pitman Adv. Publ. Progr., Boston, 1983 
[7] Ya. B. Zel' dovich and Yu. P. Raizer, Physics of Shock Waves and High Temperature Hydrodynamics Phenomena, Academic Press, New York, 1966

[8] J. Buckmaster, Viscous sheets advancing over dry beds, J. Fluid Mech. 81, 735-756 (1977)

[9] H. E. Huppert, The propagation of two-dimensional viscous gravity currents over a rigid horizontal surface, J. Fluid Mech. 121, 43-58 (1982)

[10] J. Gratton and F. Minotti, Self similar viscous gravity currents: Phase plane formalism, J. Fluid. Mech. 210, 155-182 (1990)

[11] G. J. Pert, A class of similar solutions of the non-linear diffusion equation, J. Phys. A 10, 583-593 (1977)

[12] R. E. Marshak, Effect of radiation on shock wave behavior, Phys. Fluids 1, 24-29 (1958)

[13] E. W. Larsen and G. C. Pomraning, Asymptotic analysis of non-linear Marshak waves, SIAM J. Appl. Math. 39, 201-212 (1980)

[14] G. I. Barenblatt, On the approximate solution of problems of uniform unsteady filtration in a porous media, Prikl. Mat. Mekh. 18, 351-370 (1954)

[15] G. I. Barenblatt and Ya. B. Zel' dovich, On the dipole-type solution in problems of unsteady gas filtration in the polytropic regime, Prikl. Mat. Mekh. 21, 718-720 (1957)

[16] R. E. Pattle, Diffusion from an instantaneous point source with a concentration dependent coeffcient, Quart. J. Mech. Appl. Math. 12, 407-409 (1959)

[17] R. E. Grundy, Similarity solutions of the non-linear diffusion equation, Quart. Appl. Math. 37, 259-280 (1979)

[18] G. I. Barenblatt, Similarity, Self-Similarity, and Intermediate Asymptotics, Consultants Bureau, New York, 1979

[19] D. G. Aronson, The Porous Medium Equation, Nonlinear Diffusion Problems, Lecture Notes in Math., vol. 1224, Springer-Verlag, 1986

[20] I. G. Kevrekidies, A numerical study of global bifurcations in chemical dynamics, AIChE J. 33, 1850-1864 (1987)

[20] G. Guderley, Starke Kugelige und Zylindrische Verdichtungstösse in der Nähe des Kugelmittelpunktes bzw. der Zylinderachse, Luftfahrtforschung 19, 302-312 (1942)

[21] J. A. Diez, J. Gratton, and F. Minotti, Autosimilaridad de segunda especie: Difusión hacia un centro de simetria, Internal Report, Universidad Nacional del Centro de la Provincia de Buenos Aires, 1989 (Copies are available on request)

[22] J. A. Diez, R. Gratton, and J. Gratton, Verificación experimental de una solución autosimilar de segunda especie: Flujo de lubricación convergente, Anal. AFA 1, 161-163 (1989) 